\title{
Article
}

\section{Development of an Orthogonal Tie2 Ligand Resistant to Inhibition by Ang2}

\author{
Eyad Issa, Andrew J. Moss, Marlies Fischer, Mandeep Kang, Sultan Ahmed, \\ Hussein Farah, Neil Bate, Despoina Giakomidi, and Nicholas PJ Brindle
}

Mol. Pharmaceutics, Just Accepted Manuscript • DOI: 10.1021/acs.molpharmaceut.8b00409 • Publication Date (Web): 23 Jul 2018

Downloaded from http://pubs.acs.org on July 26, 2018

\section{Just Accepted}

"Just Accepted" manuscripts have been peer-reviewed and accepted for publication. They are posted online prior to technical editing, formatting for publication and author proofing. The American Chemical Society provides "Just Accepted" as a service to the research community to expedite the dissemination of scientific material as soon as possible after acceptance. "Just Accepted" manuscripts appear in full in PDF format accompanied by an HTML abstract. "Just Accepted" manuscripts have been fully peer reviewed, but should not be considered the official version of record. They are citable by the Digital Object Identifier (DOI®). "Just Accepted" is an optional service offered to authors. Therefore, the "Just Accepted" Web site may not include all articles that will be published in the journal. After a manuscript is technically edited and formatted, it will be removed from the "Just Accepted" Web site and published as an ASAP article. Note that technical editing may introduce minor changes to the manuscript text and/or graphics which could affect content, and all legal disclaimers and ethical guidelines that apply to the journal pertain. ACS cannot be held responsible for errors or consequences arising from the use of information contained in these "Just Accepted" manuscripts. 


\title{
Development of an Orthogonal Tie2 Ligand
}

\author{
Resistant to Inhibition by Ang2
}

\author{
Eyad Issał, Andrew J. Mossł, Marlies Fischerł, Mandeep Kang, Sultan Ahmed, Hussein Farah, \\ Neil Bate, Despoina Giakomidi and Nicholas PJ Brindle*
}

University of Leicester Department of Cardiovascular Sciences and Department of Molecular and Cell Biology, Henry Wellcome Building, Lancaster Road, Leicester LE1 7RH UK

†These authors contributed equally to this work

${ }^{*}$ Corresponding Author

Nicholas PJ Brindle

Dept of Molecular \& Cell Biology \& Dept of Cardiovascular Sciences

University of Leicester

Henry Wellcome Building

Lancaster Road

Leicester LE1 7RH UK

Email:npjb1@le.ac.uk

Tel: +44(0) 1162297170 
Fax: +44(0) 1162297018

\section{ABSTRACT}

Angiopoietin-1 (Ang1) is a vascular protective ligand that acts through the receptor tyrosine kinase Tie2 to enhance endothelial survival and quiescence. In sepsis, diabetic retinopathy, and a range of other diseases, Ang2, an antagonist of Tie2, increases markedly. This antagonist suppresses Ang1 protective effects leading to vascular destabilization, inflammation and endothelial death. Administration of recombinant Ang1 can counter Ang2 antagonism and restore vascular function. However, recombinant Ang1 is needed at sufficiently high concentrations to block Ang2, and the protein is difficult to produce, requires mammalian expression systems and is prone to aggregation. Here we present an engineered synthetic Tie2 ligand that is not antagonised by Ang2, easy to produce and more robust than Ang1. Using peptide phage display we isolated a heptameric sequence that binds Tie2 ectodomain, and fused this to the coiled:coil domain of cartilage oligomeric matrix protein. This pentameric protein is $60 \mathrm{kDa}$ in size, expressed in E. coli and facile to purify. The protein, designated TSL1, binds to Tie2 ectodomain in vitro and on the cell surface. TSL1 inhibits endothelial apoptosis. Crucially TSL1 binds at a site on Tie2 distinct from the angiopoietin binding site and is resistant to antagonism by Ang2. This engineered ligand has several advantages over recombinant Ang1 for potential therapeutic applications. The study also highlights the value of orthogonal ligands for regulating cellular receptors without being subject to antagonism or modulation by endogenous ligands.

Keywords: angiopoietin, endothelial, apoptosis, synthetic ligand 


\section{INTRODUCTION}

The receptor tyrosine kinase Tie 2 is expressed predominantly by endothelial cells and has essential roles in blood vessel formation and maintenance ${ }^{1-2}$. The best-characterized ligands for Tie2 are angiopoietin-1 (Ang1) and angiopoietin-2 (Ang2). Ang1 is a vascular protective ligand and it acts via Tie2 to stimulate endothelial and microvessel survival, and suppress vascular inflammation, amongst other effects ${ }^{3-5}$. Ang2 binds to the same site on the Tie2 receptor as Ang1 and can act as a context-dependent antagonist, suppressing Ang1 binding and inhibiting protective Ang1 actions ${ }^{1}$. Ang2 is markedly increased in a range of pathologies such as sepsis, diabetic retinopathy and stroke, where it can antagonize Ang1 leading to vessel regression, leakage and inflammation ${ }^{1}$. Under these conditions administration of exogenous Ang1 has been shown to prevent endothelial death and capillary loss, suppress vascular leakage and inflammation in preclinical models of stroke, sepsis, endotoxaemia and cardiac allograft vasculopathy ${ }^{6-13}$.

Ang1 has substantial potential as a biotherapeutic, however there are a number of issues that restrict its use. The ligand is a glycoprotein that exists in a variety of multimeric states, predominantly trimeric, tetrameric and higher order states ${ }^{14-15}$. Recombinant Ang1 is difficult to produce, requires mammalian expression systems, is prone to aggregation and exhibits variable bioactivity ${ }^{16}$. Furthermore, as Ang1 is needed therapeutically in conditions in which Ang2 is elevated, it must be present at concentrations sufficiently high to overcome antagonism by Ang2. Such concentrations raise the possibility of deleterious effects, such as the increased vessel remodelling in established vessels reported to occur with elevated Ang1 ${ }^{17-18}$. The potential 
biotherapeutic uses for Ang1 warrant development of Ang1-mimetics without the limitations of the natural ligand.

In this study, we have sought to exploit the mechanism by which Tie2 is activated to create a synthetic ligand that is easy to produce, stable and more robust than Ang1. We report an orthogonal ligand that, in contrast to the native ligand, is resistant to the antagonistic effects of Ang2. This new ligand may offer significant advantages over Ang1 for therapeutic applications.

\section{MATERIALS AND METHODS}

Tie2-Fc, Tie1-Fc and VEGFR2-Fc ectodomain fusion proteins were from R \& D Systems, HisGrab nickel-coated plates and protein-A-coated plates were from Pierce/Thermo Scientific, and uncoated microtitre plates were from Nunc International. Anti-M13 antibody was from GE Healthcare, anti-FLAG was from Sigma. All other reagents were as previously reported ${ }^{19-20}$.

Phage display. Identification of a peptide binding Tie 2 ectodomain was achieved by screening a 7-mer random peptide phage display library (Ph.DTM-7; New England Biolabs) for binding to recombinant His-tagged Tie2 ectodomain-Fc fusion protein. Nickel-coated 96 well microtitre wells were incubated overnight at $4^{\circ} \mathrm{C}$ with $1 \mu \mathrm{g} / \mathrm{ml}$ Tie2-Fc before washing and blocking for $1 \mathrm{hr}$ at room temperature in TBS $(50 \mathrm{mM}$ Tris $\mathrm{pH} 7.4,150 \mathrm{mM} \mathrm{NaCl})$ with $1 \%(\mathrm{w} / \mathrm{v})$-bovine serum albumin and $0.1 \%(\mathrm{v} / \mathrm{v})$ Tween-20. Phage were allowed to bind for $15 \mathrm{~min}$ at room temperature. Unbound phage were removed by washing in TBS with $0.1 \%(\mathrm{v} / \mathrm{v})$ Tween-20. Bound phage were eluted with $10 \mu \mathrm{g} / \mathrm{ml}$ soluble Tie2-Fc. The selected phage pool was amplified by propagation in E. coli and screened for a second round against Tie2-Fc immobilized on Protein A-coated microtitre plates. A third round of screening was performed with eluted and amplified 
phage against Tie2-Fc immobilized on nickel coated plates and eluted phage amplified and screened finally for binding to Tie2-Fc in solution followed by recovery of Tie2-Fc and bound phage with protein-G-sepharose. Following elution clones were sequenced.

Ligand construction and expression. cDNA encoding the coiled:coil domain of rat cartilage oligomeric matrix protein, COMP, (DLAPQMLRELQETNAALQDVRELLRQQVKEITFLKNTVMECDACG) followed by a linker comprising of GSGGPFYSHS upstream of the Tie2-binding peptide and C-terminal sequence GGGSA was constructed and inserted into an E. coli expression vector with an amino terminal FLAG-epitope tag and His6 sequence. Following transformation E. coli were induced with IPTG for $4 \mathrm{hr}$ before collection and lysis in 50mM Tris $\mathrm{pH} 7.4,150 \mathrm{mM} \mathrm{NaCl}, 5 \%$ glycerol, $10 \mathrm{mM}$ imidazole, $0.5 \mathrm{mM}$ dithiothrietol (DTT) and protease inhibitor (Complete Protease Inhibitor; Roche). After clearing by centrifugation expressed proteins were purified by affinity chromatography on nickel columns and dialysed extensively against TBS as described previously ${ }^{21}$. Ligand concentrations were calculated using the molecular mass of the pentameric ligand. Analytical size exclusion chromatography was performed using a Superdex-200 (10/30) column (GE Healthcare) pre-equilibrated and run with TBS.

ELISA binding assays. Phage binding assays were performed by coating microtitre plates with ectodomain-Fc fusion proteins at $10 \mu \mathrm{g} / \mathrm{ml}$, washing with TBST (TBS with $0.1 \%(\mathrm{v} / \mathrm{v})$ Tween20) and blocking in TBST containing $10 \mathrm{mg} / \mathrm{ml}$ bovine serum albumin. Phage $\left(1.26 \times 10^{8} /\right.$ well $)$ were allowed to bind for $1 \mathrm{~h}$ at room temperature before extensive washing followed by detection of bound phage with anti-M13 antibody. Ligand binding ELISA assays were performed with ectodomain-Fc coated ELISA plates using a similar method and ligand binding was detected with anti-FLAG antibody. For competition binding ELISA, plates were coated with Tie2-Fc and, 
following washing and blocking, $0.2 \mathrm{nM}$ biotinylated-Ang2 was allowed to bind, alone or in the presence of 4nM Ang1 or 170nM TSL1, before extensive washing followed by detection of bound Ang2 with streptavidin conjugated to peroxidase.

Cellular assays. Human umbilical vein endothelial cells (HUVEC) were obtained from Promocell and were cultured in Medium 200 with low serum growth supplement and 10\% (v/v) foetal bovine serum.

Ligand immunoprecipitation was performed as previously described ${ }^{22}$. Briefly cells were incubated with the ligand for 15 min before addition of the cell-impermeable crosslinker 3,3'dithiobis(sulfosuccinimidylpropionate) (DTSSP) to a final concentration of $0.5 \mathrm{mM}$ in PBS on ice for 30 min. Cross-linking was quenched with $20 \mathrm{mM}$ Tris in PBS, cells were the washed with PBS and lysed in lysis buffer $(50 \mathrm{mM}$ Tris $\mathrm{pH} 7.4,50 \mathrm{mM} \mathrm{NaCl}, 1 \mathrm{mM}$ sodium fluoride, $1 \mathrm{mM}$ EGTA, 1mM sodium orthovanadate, 1\% TritonX-100). For immunoprecipitation, lysates containing equal amounts of protein were pre-cleared by incubation with protein-G agarose for $30 \mathrm{~min}$, centrifuged at $13,000 \mathrm{~g}$ for $5 \mathrm{~min}$ and supernatants removed and immunoprecipitated by addition of $2 \mu \mathrm{g}$ of anti-His 6 antibody for $2 \mathrm{hr}$ in the presence of protein-G-agarose. Immunoprecipitates were recovered by centrifugation and washed three times with wash buffer (as lysis buffer but with $0.1 \%$ Triton X100). Proteins were eluted by addition of Laemmli sample buffer containing $100 \mathrm{mM}$ DTT and boiled for $5 \mathrm{~min}$ before resolving by polyacrylamide gel electrophoresis (SDS-PAGE) followed by immunoblotting. Tie2 phosphorylation was examined by immunoblotting as previously described ${ }^{22}$. Briefly, cells were placed in serum-free medium for one hour before stimulation for $30 \mathrm{~min}$. Cells were transferred to ice, washed with ice-cold PBS and lysed in ice-cold lysis buffer. Lysates were cleared by centrifugation and assayed for protein content before resolving proteins by SDS-PAGE followed by immunoblotting. Blots 
were probed with anti-phosphoTie2(pY992) from R \& D Systems, stripped and reprobed with anti-Tie2. Bands on immunoblots were quantified by densitometric scanning of films.

Endothelial survival was determined by quantifying viable cell numbers following serumdeprivation. Briefly equal numbers of HUVEC were plated into wells of a 24 well plate and cultured for $24-48 \mathrm{hr}$ in complete growth medium. After this time medium was replaced with serum- and growth supplement-free medium containing the ligands indicated in Results. Cells were cultured for $24 \mathrm{hr}$, the medium was collected and floating cells recovered by centrifugation. Numbers of dead cells in the medium was quantified by counting trypan blue positive nuclei. Remaining adherent cells were washed twice with PBS, trypsinized, collected by centrifugation and viable cell numbers were quantified by counting cells excluding $0.2 \%(\mathrm{w} / \mathrm{v})$ trypan blue.

In some experiments cells were analysed by flow cytometry for Annexin-V binding. In this case at the end of the serum-deprivation period the culture medium was removed from each well and adherent cells recovered by trypsinization and resuspension in complete growth medium. Harvested cells were combined with culture medium supernatant from the relevant well (containing detached cells), collected by centrifugation and resuspended cells incubated with Annexin-V-FITC and propidium iodide at room before transferring to ice. Cells were analysed on a BD FACSCanto II. 


\section{RESULTS AND DISCUSSION}

Ang1 has significant potential as a therapeutic for suppression of microvessel regression and vascular dysfunction in a wide range of diseases, as well as use in tissue engineering, regenerative medicine and therapeutic angiogenesis. Despite promising pre-clinical data there are problems associated with use of the ligand. Recombinant Ang1 is difficult to produce, has been reported to have variable bioactivity and stability, tends to aggregate and has a high molecular mass of more than $280 \mathrm{kDa}$ in its active form ${ }^{16}$. Furthermore, exogenous Ang1 must be provided at high enough concentrations to overcome the antagonistic actions of Ang2 if it is to be useful in diseases associated with elevated levels of Ang2. There have already been attempts to create improved Ang1-mimetic materials. An engineered form of Ang1, COMP-Ang1, has been reported in which the receptor-binding domain of Ang1 has been fused to the COMP coiled:coil domain ${ }^{16}$. This ligand has much better stability and more consistent bioactivity ${ }^{16}$. However, like natural Ang1, COMP-Ang1 requires a mammalian expression system. Furthermore, COMPAng1 binds the same site on Tie2 as Ang2 and therefore is subject to antagonism by Ang2. An alternative to modifying natural Ang1 is the recent report of a Tie 2 agonistic antibody ${ }^{23}$. This antibody appears to be a promising alternative to both the natural ligand and recombinant variants.

In the present study, we sought to develop an orthogonal ligand for Tie2 that could be used therapeutically and would offer advantages over the natural ligand Ang1. Specifically, we aimed to create a ligand that was more robust and easier to produce. In common with other receptor tyrosine kinases, Tie 2 is activated by ligand-induced oligomerization ${ }^{14}$. For Tie2 in endothelial cells the minimal oligomerization state for receptor activation by Ang1 is a tetramer ${ }^{14}$. We aimed 
to exploit this mechanism of receptor activation to create a ligand that could bind and oligomerize Tie2, thereby inducing receptor activation. The design of the ligand is shown schematically in Fig 1B and consists of a receptor-binding peptide attached to a flexible linker and oligomerization scaffold.

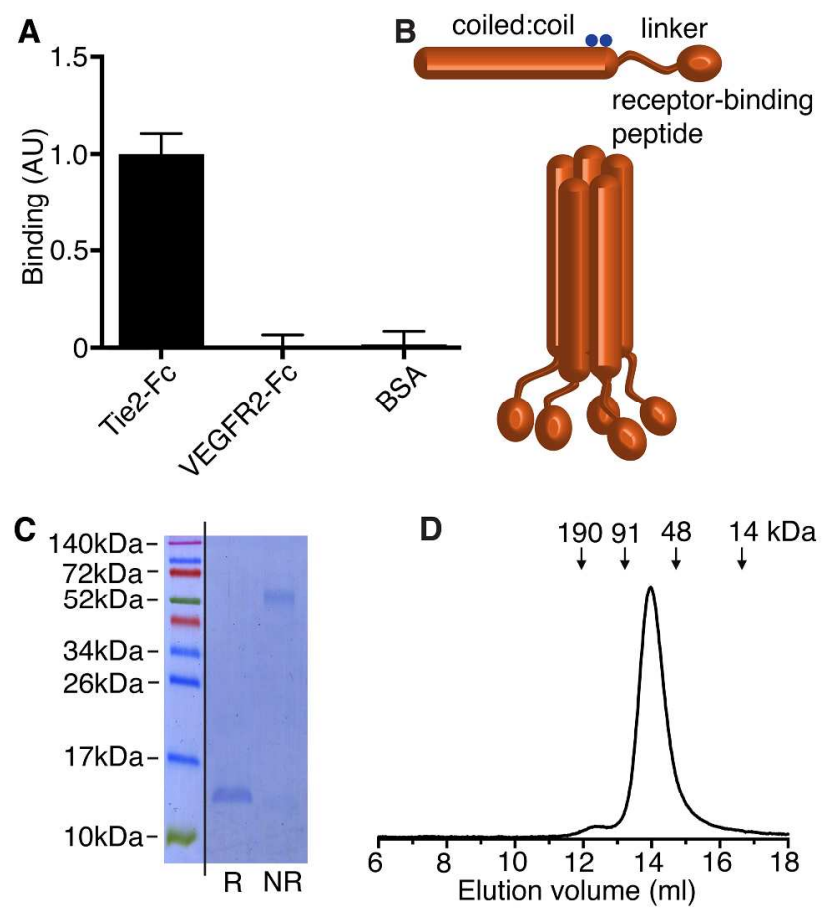

Figure 1. Tie2 synthetic ligand. (A) Binding of the peptide-displaying phage to Tie2-Fc. The ability of the phage selected by Tie2 screening to bind Tie2-Fc, VEGFR2-Fc and BSA was determined by ELISA. Data are normalized to Tie2-Fc binding and shown for three independent experiments as means and standard error of means. (B) Schematic design of synthetic ligand comprising the receptor binding peptide fused via a flexible linker to the coiled:coil domain of cartilage oligomeric matrix protein. The pentameric form is also shown schematically. Approximate positions of the cysteine residues involved in stabilizing the pentameric structure are indicated by blue circles in the monomer. (C) Monomeric and pentameric forms of expressed 
and purified TSL1. TSL1 was resolved by SDS-PAGE under non-reducing (NR) and reducing R) conditions and detected by Coomassie staining. Molecular mass marker lane from same gel shown, tracks between markers and TSL1 removed (indicated by line), the masses of some markers are indicated in kDa. Under non-reducing conditions TSL1 is seen in its pentameric $60 \mathrm{kDa}$ form whereas under reducing conditions the monomeric $12 \mathrm{kDa}$ form predominates. (D) Analytical size exclusion chromatography was performed on purified TSL1. Elution profile of TSL1 is shown and the positions of protein size markers run under same conditions are indicated in $\mathrm{kDa}$.

Receptor-binding peptide. In order to identify peptides that bind the Tie2 extracellular domain, a random 7 mer peptide phage display library was screened. Four rounds of screening were performed using Tie2-ectodomain-Fc fusion protein as target. The first round screen was performed against the ectodomain immobilized on nickel-coated microtitre plates. To maximize the probability of isolating peptides that bind Tie 2 ectodomain the initial screen was followed by alternating rounds against Tie-2 immobilized on Protein-A beads and then nickel-coated plates and finally Tie2-Fc in solution followed by recovery with Protein-G-beads. Sequencing revealed one peptide, with the sequence VTSRGNV, that accounted for more than $70 \%$ of binding clones. To confirm phage-VTSRGNV recognizes Tie2 ectodomain and that binding is not to the Fc moiety of Tie2-Fc, binding to Tie2-Fc and vascular endothelial growth factor receptor-2-Fc (VEGFR2-Fc) was compared (Fig. 1A). The phage-VTSRGNV bound to Tie2-Fc but did not bind VEGFR2-Fc above background levels. The VTSRGNV peptide sequence is not present in the natural angiopoietin ligands. 
Ligand Construction. Activation of Tie2 in endothelial cells requires the receptor to form tetrameric or higher order oligomers ${ }^{14-15}$. The receptor-binding peptide was therefore incorporated into an oligomerization scaffold (Fig 1B). The coiled:coil domain of rat cartilage oligomeric matrix protein (COMP) was used as an oligomerization scaffold. The COMP coiled:coil domain forms a stable pentameric structure ${ }^{24}$ and has previously been used as a scaffold for presenting the receptor-binding domain of the natural Tie2 ligand Ang1 to the receptor $^{16}$ and to create other pentameric fusion proteins ${ }^{25}$. In order to allow flexibility for the peptide to bind Tie2, and not to restrict the interaction between Tie2 protomers bound to the peptide, a flexible linker was incorporated between the COMP-domain and the peptide.

The constructed ligand was expressed as a soluble protein in E. coli and purified. The calculated molecular mass of the monomeric ligand is $11.9 \mathrm{kDa}$ and the purified ligand migrated as a single band of approximately $12 \mathrm{kDa}$ in SDS-PAGE under denaturing conditions in the presence of DTT (Fig. 1C). The coiled:coil domain of COMP is able to retain its pentameric structure in SDS-PAGE in the absence of $\mathrm{DTT}^{16}$. This high stability of COMP coiled:coil domain is due to a ring of disulphide bonds formed between cysteine residues in the carboxytermini of the domains (Cys71 of one chain and Cys68 of the neighbouring chain in rat COMP) ${ }^{24,}$ 26-27. When the ligand was resolved by SDS-PAGE in the absence of DTT it was present as a $60 \mathrm{kDa}$ band, corresponding to the pentamerized ligand (Fig. 1C). Analytical size exclusion chromatography under native conditions shows the purified protein predominantly as a single species of approximately 60kDa with no evidence of higher order aggregates (Fig. 1D). The fusion protein was designated Tie2-synthetic ligand-1 (TSL1). 
Ligand Binding. ELISA was performed with the constructed ligand to confirm its ability to bind Tie2 ectodomain. As shown in Figure 2A, TSL1 bound Tie2 immobilized on ELISA plates in a concentration-dependent manner. Half-maximal binding was achieved at $18.9+/-1.5 \mathrm{nM}$ TSL1 (mean +/- SEM, n=3). For comparison, half maximal binding of Ang1 to Tie2 measured by ELISA under similar conditions was achieved at $0.14+/-0.04 \mathrm{nM}$ Ang1 (mean +/- SEM, n=3, molar concentration assumes tetrameric Ang1) ${ }^{21}$ indicating higher concentrations of TSL1 compared to Ang1 are required to achieve half maximal binding to Tie2. We also examined binding selectivity of TSL1 for Tie1-Fc and VEGFR2-Fc and found clear selectivity for Tie2 (Fig. 2B), as predicted from phage-displayed peptide binding (Fig. 1A). However, there was also detectable binding to Tie1, with $20 \%$ of level of Tie2 binding at a TSL1 concentration of 100nM (Fig. 1A). 

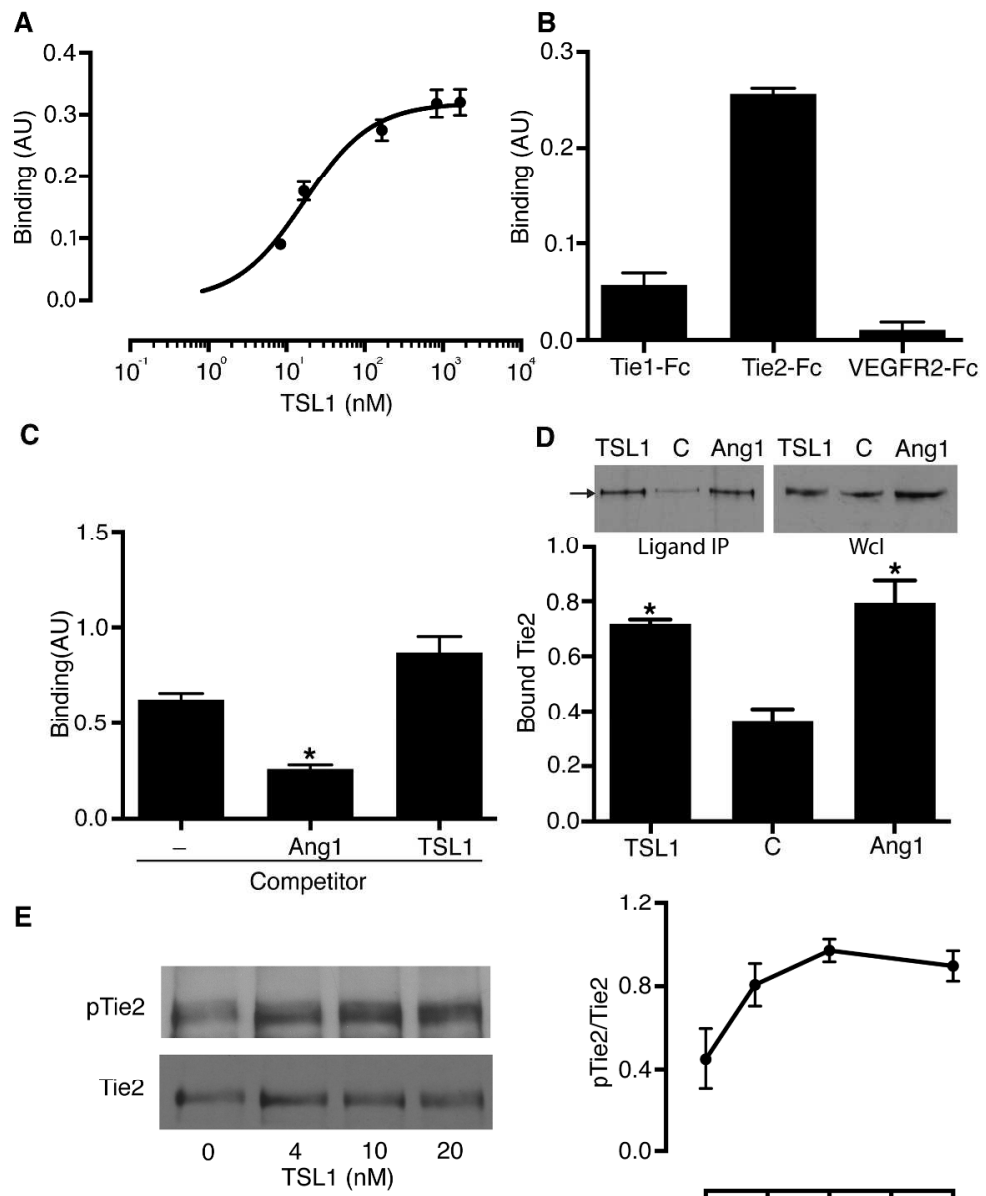

25 E

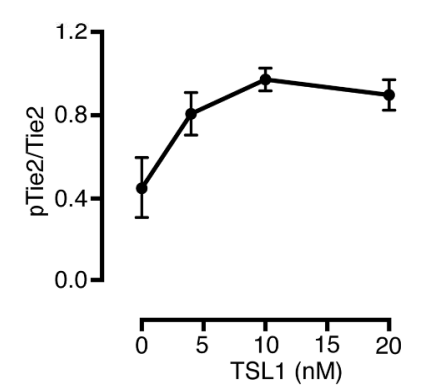

Figure 2. TSL1 binds Tie2. (A) Concentration dependent binding of TSL1 to Tie-Fc. Purified TSL1 was tested for binding to immobilised Tie2 by ELISA. Data are shown as means and standard deviation for triplicate determinations from a single experiment representative of three independent experiments. (B) Selectivity of TSL1 for Tie2. TSL1 at 100nM was assayed for binding to immobilized Tie1-Fc, Tie2-Fc and VEGFR2-Fc by ELISA, data are shown for three independent experiments as means and standard error of the means. (C) Competition with Ang2 for binding to Tie2. ELISA plates were coated with Tie2-Fc and the ability of $0.2 \mathrm{nM}$ biotinylated Ang2 to bind was tested in the absence or presence of 4nM Ang1 or 170nM TSL1, as indicated. Data are shown for three independent experiments as means and standard error of the means. 
Ang1 inhibits Ang2 binding (*, p $<0.05$, one way ANOVA followed by Sidak's multiple comparisons test). (D) TSL1 binds cellular Tie2. HUVEC were incubated with 10nM TSL1, control or 1nM Ang1 followed by cross-linking and recovery of ligand by immunoprecipitation with anti-His ${ }_{6}$ antibody. Immunoprecipitates were resolved by SDS-PAGE and bound Tie2 detected by immunoblotting (ligand IP), and input cell lysates were probed in parallel for Tie2 (Wcl). Blots from a representative experiment is shown and the position of Tie2 is indicated with an arrow. Bound Tie2 recovered by TSL1, Ang1 or control IP was quantified by densitometric scanning of IP blots and normalized to the relative levels of Tie2 present in whole cell lysate blots for three independent experiments and is presented in the bar graph. Both TSL1 and Ang1 bound more cellular Tie2 than the negative control background IP $(*, p<0.05$, one way ANOVA followed by Sidak's multiple comparisons test). (E) TSL1 stimulates Tie2 phosphorylation. Endothelial cells were stimulated with TSL1 as indicated and tyrosine phosphorylated Tie2 detected by immunoblotting. Blots were then re-probed for total Tie2. A representative blot is shown. Phosphorylated Tie2 was quantified by densitometric scanning of blots and normalized to the relative levels of Tie2 for three independent experiments and is presented in graphical form.

In order to test for competition between TSL1 and natural Tie2 ligands, competition ELISA was performed in which biotinylated-Ang2 binding was assessed in the absence and presence of Ang1 and TSL1. Ang1 suppressed binding of Ang2 to Tie2 (Fig. 2C), consistent with Ang1 and Ang2 binding to the same site on Tie2. In contrast, TSL1 at approximately 1000-fold molar excess did not affect Ang2 binding to Tie2 (Fig. 2C), indicating TSL1 does not compete with Ang2 for binding to Tie2. 
In addition to examining Tie2 binding in vitro the ability of TSL1 to bind Tie 2 was assessed on endothelial cells. To do this TSL1 was incubated with endothelial cells and the cell impermeable thiol-cleavable cross-linking agent DTSSP added. Following blocking of the cross-linker, cells were lysed and TSL1 was immunoprecipitated. Immunoprecipitates were resolved by SDSPAGE and immunoblots probed for the presence of Tie2. As seen in Figure 2D, TSL1 immunoprecipitated from endothelial cells was bound to Tie2. As a positive control, ligand immunoprecipitation from endothelial cells was also performed for Ang1 and, as expected, Ang1 bound Tie 2 on the endothelial cells. In contrast, a fusion protein identical to TSL1 but lacking the Tie2 binding peptide sequence only showed low-level background binding to Tie2, and both TSL1 and Ang1 bound to cellular Tie2 above the level of background control IP (Fig. 2D). The effects of TSL1 on activation of Tie2 was examined in endothelial cells by analysing phosphorylation of Tie2 on tyrosine992 (Fig. 2E). This tyrosine residue is in the activation loop of Tie 2 and its phosphorylation correlates with stimulation of the receptor tyrosine kinase activity $^{28}$. TSL1 stimulated Tie2 phosphorylation indicating the ligand activates Tie2 signalling (Fig. 2E).

Functional Activity of Orthogonal Ligand. An important cellular effect of the natural Tie2 agonist, Ang1, is the promotion of endothelial cell survival ${ }^{4}$. The effects of TSL1 on cell survival were therefore tested. Endothelial cells were serum-deprived in the absence or presence of different concentrations of TSL1 for 24 hours and surviving cells quantified. In the presence of TSL1 endothelial survival was increased in a concentration-dependent manner (Figure 3A). Maximal effects of TSL1 were observed at 10nM TSL1, with numbers of viable cells similar to 
those seen with the natural ligand Ang1 (Figure 3A). HUVEC undergoing apoptosis are released from adhesion and appear in the culture medium ${ }^{29}$. To confirm effects of TSL1 therefore we also collected the culture medium and quantified the number of dead cells (trypan blue positive nuclei) and confirmed that TSL1 decreased endothelial cell death (Figure 3B). In addition we examined endothelial cells for the appearance of the apoptotic cell surface marker phosphatidylserine by annexin-V staining and flow cytometry (Fig. 3C). Consistent with its effects on endothelial survival following serum-deprivation, and cell death, TSL1 decreased the percentage of apoptotic endothelial cells (Fig. 3C).
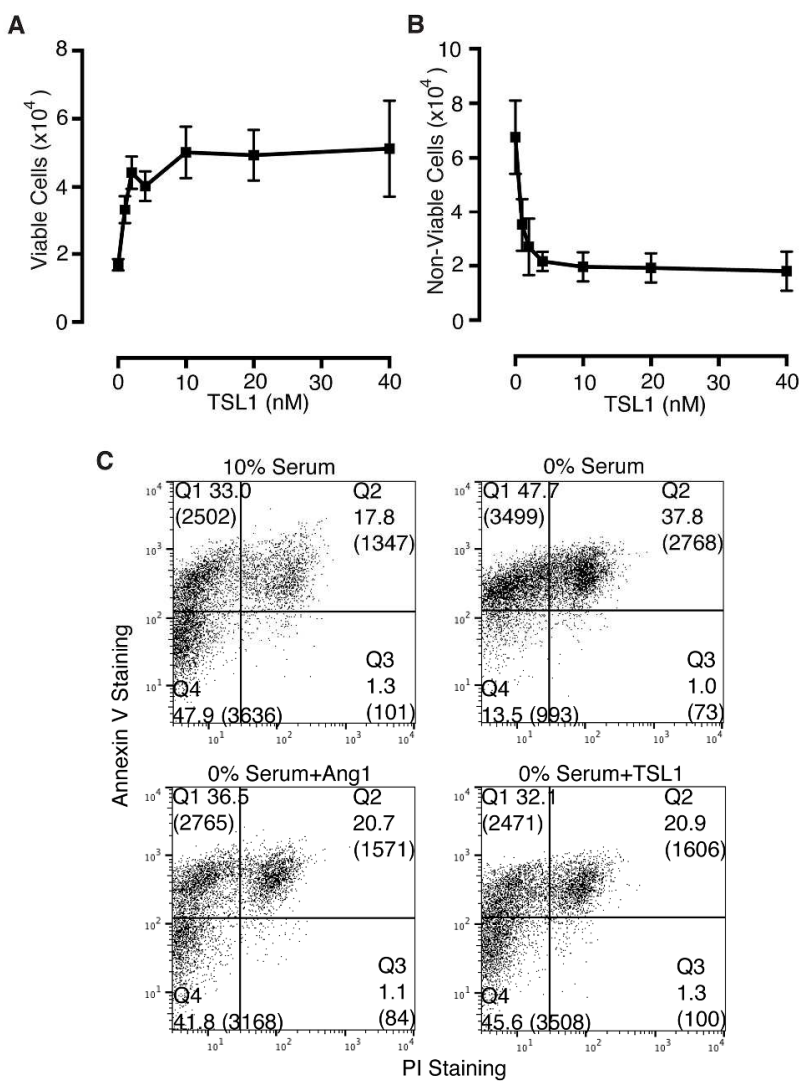

Figure 3. TSL1 inhibits endothelial cell death. Equal numbers of endothelial cells were plated into tissue culture wells, cultured in complete medium for 24-48 hr before serum deprivation in 
the presence of different concentrations of TSL1 for $24 \mathrm{hr}$ and determination of numbers of viable adherent cells (A) and non-viable cells in the media (B). Data are shown for five independent experiments as means and standard error of the means. (C) TSL1 inhibits endothelial apoptosis. Endothelial cells were maintained in $10 \%$ serum or serum deprived in the absence or presence of 10nM TSL1 or $0.18 \mathrm{nM} \mathrm{Ang} 1$ for $24 \mathrm{hr}$, as indicated. Adherent cells were collected by trypsinization, combined with floating cells, stained with annexin- $\mathrm{V}$ and propidium iodide and analysed by flow cytometry. Plots show percentage of cells in each quandrant and absolute cell numbers in parentheses. Plots show serum deprivation decreases the proportion of viable cells (Q4) and increases early and late apoptotic cells (Q1 and Q2) whereas TSL1 and Ang1 increase the fraction of viable cells and decrease apoptosis compared to serum deprivation.

TSL1 is resistant to inhibition by Ang2. Ang2 binds the same site on Tie2 as the natural agonist Ang1 and by competing with Ang1 can supress its activity. Exogenous Ang1 must be provided at high enough concentrations to overcome the antagonistic actions of Ang2 if it is to be useful at sites of new vessel formation or in diseases in which Ang2 is elevated. Unfortunately, high levels of Ang1 have significant side effects and have been shown to cause remodelling of established vessels ${ }^{17-18}$. A Tie2 agonist that is not inhibited by Ang2 could therefore potentially be used at lower concentrations decreasing the likelihood of such side effects. TSL1 and Ang2 do not compete for binding on Tie2 (Fig. 2C), therefore it is possible that Ang2 would not be able to block TSL1 action. To test this, endothelial cells were subjected to serum-deprivation and the effects of Ang2 on Ang1- and TSL1-induced endothelial viability examined. As shown in Figure 4A, Ang1 increased the number of viable endothelial cells and Ang2 inhibited this effect, consistent with the ability of Ang2 to antagonize the effects of the 
2

3

4

5

6

7

8

9

10

11

12

13

14

15

16

17

18

19

20

21

22

23

24

25

26

27

28

29

30

31

32

33

34

35

36

37

38

39

40

41

42

43

44

45

46

47

48

49

50

51

52

53

54

55

56

57

58

59

60 natural ligand. In contrast, the TSL1-induced increase in viable endothelial cells was not inhibited by Ang2 (Fig. 4A). This ability of TSL1 to escape inhibition by Ang2 may be useful in situations where Ang2 is elevated. 

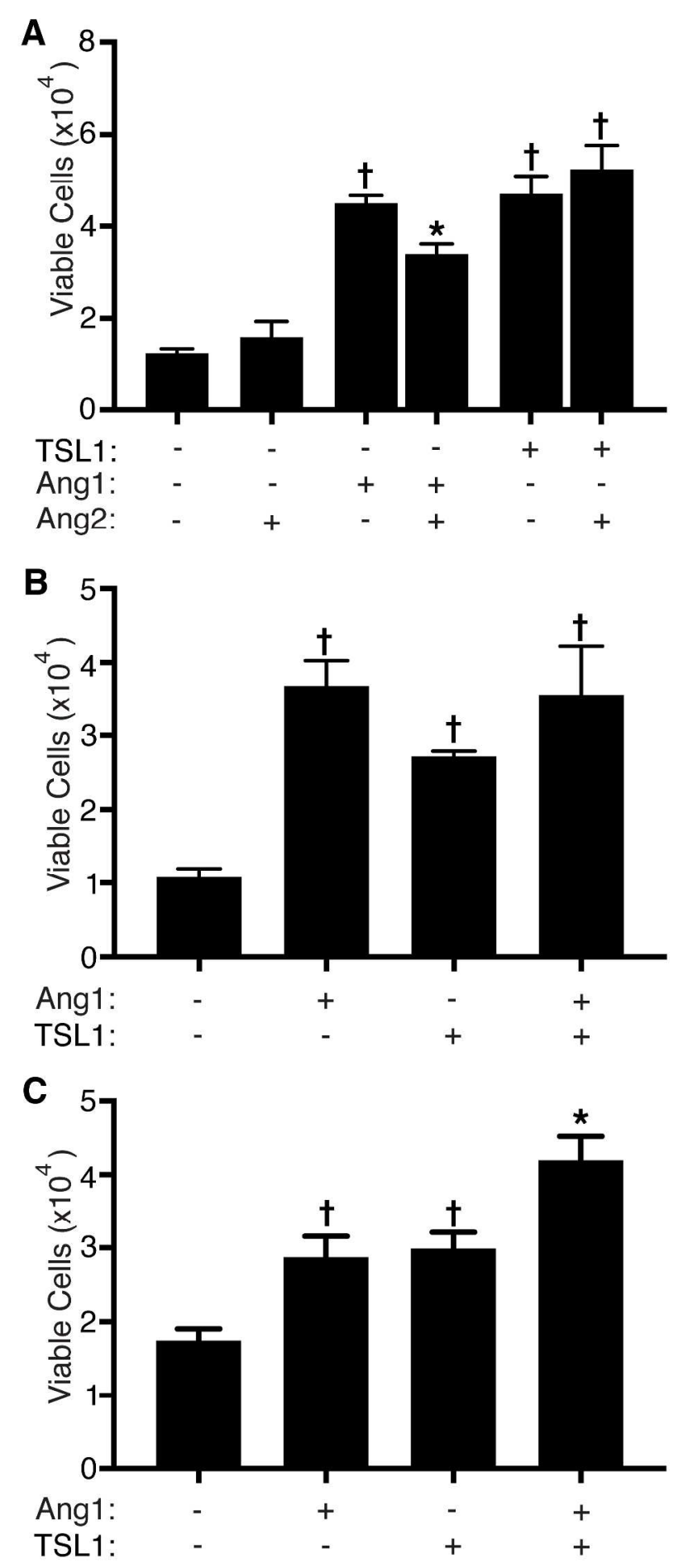

Figure 4. TSL1 acts independently of angiopoietins to promote endothelial survival. (A) Ang2 does not inhibit TSL1-induced endothelial survival. Equal numbers of endothelial cells were seeded into tissue culture plates and grown for 24-48 $\mathrm{hr}$ before incubating in serum-free 
medium in the absence or presence of $10 \mathrm{nM}$ TSL1, $0.18 \mathrm{nM}$ Ang1 or 1.4nM Ang2 as indicated. After $24 \mathrm{hr}$ the viable cell number was determined. Data are shown for four independent experiments as means and standard error of the means. Ang1 and TSL1 stimulate cell survival $(\dagger, \mathrm{p}<0.05)$ and Ang2 inhibits Ang1-induced cell survival $(*, \mathrm{p}<0.05)$, but does not affect cell survival in the presence of TSL1. There was no statistically significant difference between Ang1 and TSL1 on cell survival. (B) TSL1 does not affect Ang1-induced endothelial survival. Viable endothelial cells were quantitified following $24 \mathrm{hr}$ incubation in serum-free medium in the absence or presence of Ang1 or TSL1 as indicated. Data are shown for three independent experiments as means and standard error of the means. Ang1 and TSL1 stimulate cell survival $(\dagger, \mathrm{p}<0.05)$. There was no statistically significant difference between Ang1 and TSL1 on cell survival. (C) Additive effects of Ang1 and TSL1. Viable endothelial cells were quantitified following $24 \mathrm{hr}$ incubation in serum-free medium in the absence or presence of $0.07 \mathrm{nM}$ Ang1 or $0.85 \mathrm{nM}$ TSL1 as indicated. Data are shown for three independent experiments as means and standard error of the means. Ang1 and TSL1 stimulate cell survival $(\dagger, \mathrm{p}<0.05)$, and in the presence of both ligands cell survival is higher than Ang1 or TSL1 alone $(*, p<0.05)$. Data were analysed by one way ANOVA followed by Sidak’s multiple comparisons test.

We also examined the effects of both TSL1 and Ang1 together on endothelial survival. As shown in Figure 4B, both Ang1 and TSL1 induced increases in viable endothelial cell numbers. Addition of TSL1 together with Ang1 induced an increase in viable endothelial cells to a level similar to Angl alone, importantly there was no diminution in viable cell number when both ligands were present together. At low concentrations Ang1 and TSL1 exhibited an additive effect on viable cell numbers (Fig. 4C). 


\section{CONCLUSION}

Here we describe a novel orthogonal ligand for Tie2 that is distinct from the natural angiopoietin ligands. TSL1 binds Tie2 in vitro and at the cell surface. Furthermore TSL1 stimulates endothelial survival to a similar level as Ang1. Tie2 appears to exist mainly as preformed dimers on the endothelial surface ${ }^{30-31}$ and is activated by Ang1-induced clustering into tetrameric or higher order complexes. As TSL1 comprises five Tie2 binding peptides on a pentameric scaffold it is likely that this engineered ligand also acts to cluster Tie2 leading to receptor activation and increased cell survival. A model of how TSL1 could activate Tie2 is depicted schematically in Figure 5. Tie2 has been reported to interact with other cell surface proteins including the related receptor Tie1 and integrins such as $\alpha 5 \beta 1$, and these interactions are thought to regulate Tie 2 and/or participate in Tie 2 signalling ${ }^{32-33}$. It will be of interest in future studies to examine the effects of TSL1 on these additional interactions of Tie2, and the implications for Tie2 regulation and signalling. It is possible that the low but detectable binding of TSL1 to Tie1 could contribute to some of the cellular activity of the ligand and additional work will address this. In addition, future work will assess the size and position of the TSL1 binding site on Tie2 and potential impact of this for TSL1 action through the receptor. 
This novel engineered ligand may be useful in situations in which it is desirable to activate Tie2, and the ligand offers several advantages compared with recombinant forms of the natural ligand. The orthogonal ligand does not require glycosylation and is easy to produce. Expression and purification of the ligand is facile and utilizes an $E$ coli expression host rather than requiring the more complex and lower yielding mammalian expression systems. Crucially the orthogonal ligand is resistant to inhibition by the natural Tie2 antagonist Ang2, allowing it to be used in situations in which Ang2 is elevated, without having to outcompete Ang2. These data highlight 
the value of developing Tie2 ligands that bind the receptor at sites distinct from natural ligands and which are therefore able to resist antagonism by Ang2.

\section{ACKNOWLEDGEMENT}

This work was supported by the British Heart Foundation (PG/13/43/30312 and $\mathrm{PG} / 15 / 65 / 3170)$. 


\section{REFERENCES}

(1) Augustin, H. G.; Young Koh, G.; Thurston, G.; Alitalo, K., Control of vascular morphogenesis and homeostasis through the angiopoietin-Tie system. Nat. Rev. Mol. Cell. Biol. $2009,10,165-177$.

(2) Peters, K. G.; Kontos, C. D.; Lin, P. C.; Wong, A. L.; Rao, P.; Huang, L.; Dewhirst, M. W.; Sankar, S., Functional significance of Tie2 signaling in the sdult vasculature. Recent Prog. Horm. Res. 2004, 59, 51-71.

(3) Kim, I.; Kim, H. G.; So, J. S.; Kim, J. H.; Kwak, H. J.; Koh, G. Y., Angiopoietin-1 regulates endothelial cell survival through the phosphatidylinositol 3'-kinase/Akt signal transduction pathway. Circ. Res. 2000, 86, 24-29.

(4) Kwak, H. J.; So, J. N.; Lee, S. J.; Kim, I.; Koh, G. Y., Angiopoietin-1 is an apoptosis survival factor for endothelial cells. FEBS Lett. 1999, 448, 249-53.

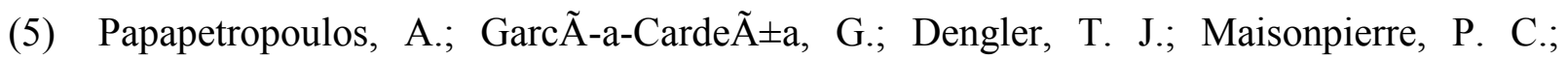
Yancopoulos, G. D.; Sessa, W. C., Direct actions of angiopoietin-1 on human endothelium: evidence for network stabilization, cell survival, and interaction with other angiogenic growth factors. Lab. Invest. 1999, 79, 213-23.

(6) Zhang, Z. G.; Zhang, L.; Croll, S. D.; Chopp, M., Angiopoietin-1 reduces cerebral blood vessel leakage and ischemic lesion volume after focal cerebral embolic ischemia in mice. Neuroscience 2002, 113, 683-7.

(7) Witzenbichler, B.; Westermann, D.; Knueppel, S.; Schultheiss, H.-P.; Tschope, C., Protective role of angiopoietin-1 in endotoxic shock. Circulation 2005, 111, 97-105. 
(8) Nykanen, A. I.; Krebs, R.; Saaristo, A.; Turunen, P.; Alitalo, K.; Yla-Herttuala, S.; Koskinen, P. K.; Lemstrom, K. B., Angiopoietin-1 protects against the development of cardiac allograft arteriosclerosis. Circulation 2003, 107, 1308-1314.

(9) Cho, C.-H.; Kammerer, R. A.; Lee, H. J.; Yasunaga, K.; Kim, K.-T.; Choi, H.-H.; Kim, W.; Kim, S. H.; Park, S. K.; Lee, G. M.; Koh, G. Y., Designed angiopoietin-1 variant, COMPAng1, protects against radiation-induced endothelial cell apoptosis. Proc. Natl. Acad. Sci. USA 2004, 101, 5553-5558.

(10) Zhao, Y. D.; Campbell, A. I. M.; Robb, M.; Ng, D.; Stewart, D. J., Protective role of angiopoietin-1 in experimental pulmonary hypertension. Circ. Res. 2003, 92, 984-991.

(11) Zhao, Y.; Li, Z.; Wang, R.; Wei, J.; Li, G.; Zhao, H., Angiopoietin 1 counteracts vascular endothelial growth factor-induced blood-brain barrier permeability and alleviates ischemic injury in the early stages of transient focal cerebral ischemia in rats. Neurol. Res. 2010, 32, 748-755.

(12) McCarter, S. D.; Mei, S. H.; Lai, P. F.; Zhang, Q.; Parker, C. H.; Suen, R. S.; Hood, R. D.; Zhao, Y. D.; Deng, Y.; Han, R. N.; Dumont, D. J.; Stewart, D. J., Cell-based angiopoietin-1 gene therapy for acute lung injury. Am. J. Respir. Crit. Care Med. 2007, 175, 1014-1026.

(13) Mei, S. H. J.; McCarter, S. D.; Deng, Y.; Parker, C. H.; Liles, W. C.; Stewart, D. J., Prevention of LPS-induced acute lung injury in mice by mesenchymal stem cells overexpressing angiopoietin 1. PLoS Med. 2007, 4 (9) e269.

(14) Davis, S.; Papadopoulos, N.; Aldrich, T. H.; Maisonpierre, P. C.; Huang, T.; Kovac, L.; Xu, A.; Leidich, R.; Radziejewska, E.; Rafique, A.; Goldberg, J.; Jain, V.; Bailey, K.; Karow, M.; Fandl, J.; Samuelsson, S. J.; Ioffe, E.; Rudge, J. S.; Daly, T. J.; Radziejewski, C.; 
Yancopoulos, G. D., Angiopoietins have distinct modular domains essential for receptor binding, dimerization and superclustering. Nat. Struct. Biol. 2003, 10, 38-44.

(15) Kim, K.-T.; Choi, H.-H.; Steinmetz, M. O.; Maco, B.; Kammerer, R. A.; Ahn, S. Y.; Kim, H.-Z.; Lee, G. M.; Koh, G. Y., Oligomerization and multimerization is critical for angiopoietin-1 to bind and phosphorylate Tie2. J. Biol. Chem. 2005, 280, 20126-20131.

(16) Cho, C.-H.; Kammerer, R. A.; Lee, H. J.; Steinmetz, M. O.; Ryu, Y. S.; Lee, S. H.; Yasunaga, K.; Kim, K.-T.; Kim, I.; Choi, H.-H.; Kim, W.; Kim, S. H.; Park, S. K.; Lee, G. M.; Koh, G. Y., COMP-Ang1: A designed angiopoietin-1 variant with nonleaky angiogenic activity. Proc. Natl. Acad. Sci. USA 2004, 101, 5547-5552.

(17) Baffert, F.; Thurston, G.; Rochon-Duck, M.; Le, T.; Brekken, R.; McDonald, D. M., Agerelated changes in vascular endothelial growth factor dependency and angiopoietin-1-induced plasticity of adult blood vessels. Circ. Res. 2004, 94, 984-992.

(18) Fuxe, J.; Lashnits, E.; O'Brien, S.; Baluk, P.; Tabruyn, S. P.; Kuhnert, F.; Kuo, C.; Thurston, G.; McDonald, D. M., Angiopoietin/Tie2 Signaling transforms capillaries into venules primed for leukocyte trafficking in airway inflammation. Am. J. Pathol. 2010, 176, 2009-2018.

(19) Tahir, T. A.; Singh, H.; Brindle, N. P., The RNA binding protein hnRNP-K mediates post-transcriptional regulation of uncoupling protein-2 by angiopoietin-1. Cell. Signal. 2014, 26, $1379-84$

(20) Singh, H.; Hansen, T. M.; Patel, N.; Brindle, N. P. J., The molecular balance between receptor tyrosine kinases Tie1 and Tie2 is dynamically controlled by VEGF and TNF $\alpha$ and regulates angiopoietin signalling. PLoS One 2012, 7 (1), e29319. 
(21) Brindle, N. P.; Sale, J. E.; Arakawa, H.; Buerstedde, J. M.; Nuamchit, T.; Sharma, S.; Steele, K. H., Directed evolution of an angiopoietin-2 ligand trap by somatic hypermutation and cell surface display. J. Biol. Chem. 2013, 288, 33205-33212.

(22) Hansen, T. M.; Singh, H.; Tahir, T. A.; Brindle, N. P., Effects of angiopoietins-1 and -2 on the receptor tyrosine kinase Tie2 are differentially regulated at the endothelial cell surface. Cell. Signal. 2010, 22, 527-32.

(23) Hwang, B.; Lee, S. H.; Kim, J. S.; Moon, J. H.; Jeung, I. C.; Lee, N. G.; Park, J.; Hong, H. J.; Cho, Y. L.; Jung, H.; Park, Y. J.; Lee, S. J.; Lee, H. G.; Kim, W. K.; Han, B. S.; Bae, K. H.; Chung, S. J.; Kwon, Y. G.; Lee, S. C.; Kim, S. J.; Min, J. K., Stimulation of angiogenesis and survival of endothelial cells by human monoclonal Tie2 receptor antibody. Biomaterials 2015, $51,119-28$.

(24) Malashkevich, V. N.; Kammerer, R. A.; Efimov, V. P.; Schulthess, T.; Engel, J., The crystal structure of a five-stranded coiled coil in COMP: a prototype ion channel? Science 1996, $274,761-5$.

(25) Matsuda, S.; Kamiya, Y.; Yuzaki, M., Roles of the N-terminal domain on the function and quaternary structure of the ionotropic glutamate receptor. J. Biol. Chem. 2005, 280 (20), 20021-9.

(26) Efimov Vladimir, P.; Lustig, A.; Engel, J., The thrombospondin $\square$ like chains of cartilage oligomeric matrix protein are assembled by a five $\square$ stranded $\alpha \square$ helical bundle between residues 20 and 83. FEBS Lett. 1994, 341, 54-58. 
(27) Efimov Vladimir, P.; Engel, J.; Malashkevich Vladimir, N., Crystallization and preliminary crystallographic study of the pentamerizing domain from cartilage oligomeric matrix protein: A five $\square$ stranded $\alpha \square$ helical bundle. Proteins: Structure, Function, and Bioinformatics $1996,24,259-262$.

(28) Murray, B. W.; Padrique, E. S.; Pinko, C.; McTigue, M. A., Mechanistic effects of autophosphorylation on receptor tyrosine kinase catalysis: enzymatic characterization of Tie2 and phospho-Tie2. Biochemistry 2001, 40, 10243-53.

(29) Levkau, B.; Scatena, M.; Giachelli, C. M.; Ross, R.; Raines, E. W., Apoptosis overrides survival signals through a caspase-mediated dominant-negative NF-kappa B loop. Nat. Cell Biol. $1999,1,227-33$.

(30) Yamakawa, D.; Kidoya, H.; Sakimoto, S.; Jia, W.; Naito, H.; Takakura, N., Ligandindependent Tie2 dimers mediate kinase activity stimulated by high dose angiopoietin-1. J. Biol. Chem. 2013, 288, 12469-77.

(31) Moore, J. O.; Lemmon, M. A.; Ferguson, K. M., Dimerization of Tie2 mediated by its membrane-proximal FNIII domains. Proc. Natl. Acad. Sci. U. S. A. 2017, 114, 4382-4387.

(32) Felcht, M.; Luck, R.; Schering, A.; Seidel, P.; Srivastava, K.; Hu, J.; Bartol, A.; Kienast, Y.; Vettel, C.; Loos, E. K.; Kutschera, S.; Bartels, S.; Appak, S.; Besemfelder, E.; Terhardt, D.; Chavakis, E.; Wieland, T.; Klein, C.; Thomas, M.; Uemura, A.; Goerdt, S.; Augustin, H. G., Angiopoietin-2 differentially regulates angiogenesis through TIE2 and integrin signaling. J. Clin. Invest. 2012, 122, 1991-2005.

(33) Saharinen, P.; Leppanen, V. M.; Alitalo, K., SnapShot: Angiopoietins and their functions. Cell 2017, 171 (3), 724-724 e1. 


\title{
For Table of Contents Use Only
}

\section{Development of an Orthogonal Tie2 Ligand}

\author{
Resistant to Inhibition by Ang2
}

Eyad Issał, Andrew J. Mossł, Marlies Fischerł, Mandeep Kang, Sultan Ahmed, Hussein Farah, Neil Bate, Despoina Giakomidi and Nicholas PJ Brindle*

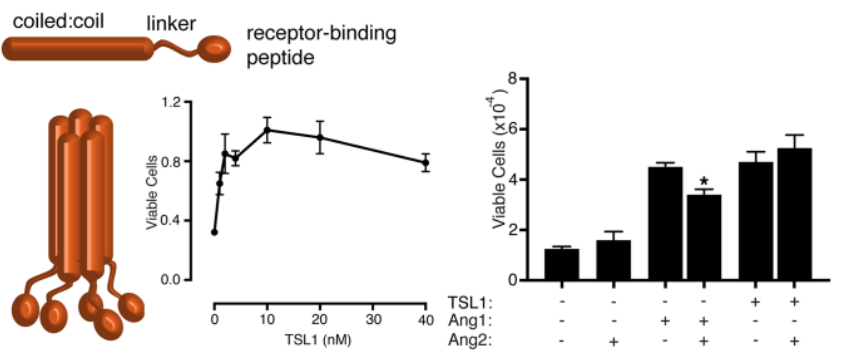

